\title{
Research on the Purchase Model of Equipment Reserves
}

\author{
Shunhua Liu ${ }^{1}$, Xuezheng Zhu ${ }^{1}$, Zhizhen Zhu ${ }^{2}$, Kunlin Nie ${ }^{1}$ \\ ${ }^{1}$ Institute of Chemical defense, ${ }^{2}$ The Military Representative Office of Chemical defense in Kunming \\ liu_shun_hua@sohu.com
}

Keywords: Equipment; Reserves; Model; Purchasing

\begin{abstract}
In order to optimize the efficiency-cost ratio, a stepwise purchase model that can finish the quota of equipment reserves within a given time is established based on the parameters of equipment life, production capacity and price, and the optimum efficiency-cost ratio of purchasing is achieved through being verified by several numerical examples, which provides a theoretical method and computational model for the decisions of equipment purchasing.
\end{abstract}

\section{Introduction}

Equipment is the material basis of various industries to complete professional tasks, which plays an important role for the usually big demand on its variety and quantity, in order to deal with the various cases and maintain the stability of completing tasks, a certain size of equipment reserves are needed. Due to the unpredictability and greater uncertainty of the future emergencies, if there are no corresponding reserves, the consequences will be unimaginable; whereas, if the inventory is too large and there is no unexpected situation, and a huge waste of funds will be caused by purchasing, maintenance, losing efficacy and scrapping. As the equipment has a certain production cycle, lifespan and purchasing costs, how to establish reserves by means of purchasing within a given time, and optimize the efficiency-cost ratio, are the problems that need to be studied and solved by all departments at present. Because of the lack of practical data, the research on equipment reserves faces great difficulties. Based on the reserve quota, how to establish the equipment reserves by stepwise purchase and achieve the optimum efficiency-cost ratio are studied in this paper.

\section{Modeling}

The demand for some kind of equipment is set to Q, the production capacity is C, when dealing with emergencies, the time can be used for production is $\mathrm{T}$, and the equipment inventory is $\mathrm{M}$, the calculation is shown in formula (1).

$$
M=Q-C T
$$

Model assumptions: the equipment reserves should be established in the prescribed time limit ( $T_{1}$ year), and the inventory remains the same, the maximum production capacity of the equipment is steady, and the lifespan is $T_{2}$, the purchase amount of the equipment in year $i$ is $q_{i}$, and there is no emergency during this period (such as war, etc.).

Model targets: the inventory reaches to the reserve quota of equipment in the prescribed time limit ( $T_{1}$ year) with the least funds, that is to get the solution of the annual optimal purchasing quantity that the equipment inventory reaches to $\mathrm{M}$ within $T_{1}$ years.

The stepwise purchase model when $T_{1}<T_{2}$.

When the purchasing time is less than the lifespan of equipment, the stepwise purchase model is established, as shown in Figure 1.

In the year $T_{1}$, the equipment inventory reaches to $M$, so the annual purchasing quantity of the equipment is 0 during $T_{1} \sim T_{2}$; $T_{2}$ years later, the equipment will scrap continually because of losing efficacy, in order to make the equipment inventory remains at the level of $M$, a certain amount of equipment should be purchased ever year. 


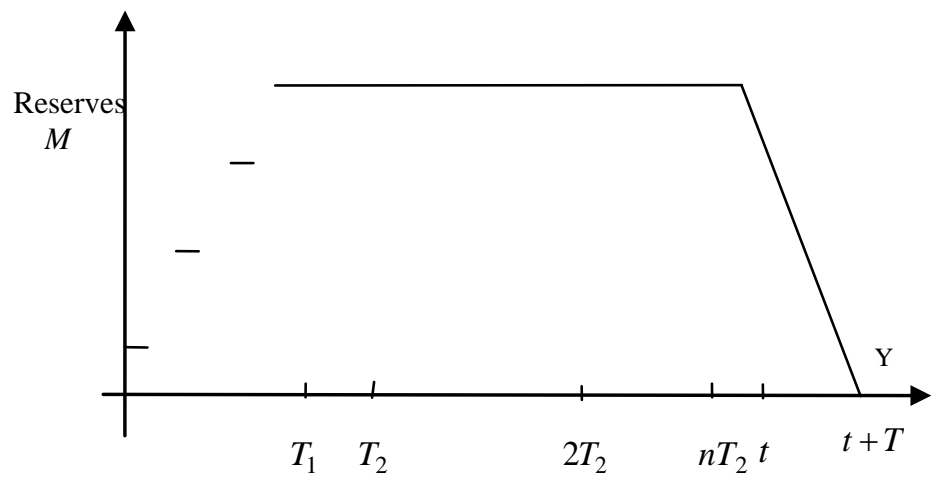

Figure 1.The relationship between the reserves and time when $T_{1}<T_{2}$

Therefore, the equipment reserves established a dynamic system with continuous inflows and outflows due to the scrapping and supplementary purchase, this system can achieve the dynamic equilibrium only when the annual purchase volume is equal to the number of the scrapped equipment of this year. So, the annual purchase quantity of equipment will repeat in every $T_{2}$ years, namely, the annual purchase quantity is a function with the period of $T_{2}, \operatorname{in}\left[0, T_{2}\right]$, see formula (2).

$$
q_{i}=\left\{\begin{array}{cc}
q_{1} & i=1 \\
q_{2} & i=2 \\
\vdots & \vdots \\
q_{T_{1}} & i=T_{1} \\
0 & T_{1}<i \leq T_{2}
\end{array}\right.
$$

When $i>T_{2}, q_{i}=q_{r}$, where $r$ is a remainder that of $i$ divided by $T_{2}$.

If an emergency occurs in the year $t$, the duration is $T$ years, if the equipment produced in the $T$ years can be put into use directly, and which will not be included in the reserves, the equipment reserves present a linear decreasing trend due to the large consumptions in $T$ years, however, if the equipment can be put into use before scrapping, the average rate of reduction in equipment reserves is $v=M / T$.

\section{1) Objective Function}

\section{a) Purchasing Costs}

For equipment, the purchase price is not only related to the purchase quantity, but also changes with time, for simplicity, the price changes with time can be ignored, and the unit price of equipment is regarded as a constant, the unit price of a certain equipment is set to $p$, the calculation for purchase cost in the first period can be seen in formula (3).

$$
O C_{1}=p q_{1}+p q_{2}+\cdots p q_{T_{1}}+p \cdot 0+\cdots p \cdot 0=p\left(q_{1}+q_{2}+\cdots+q_{T_{1}}\right)=p M
$$

The periodicity of purchase quantity shows that the purchase cost of each period is a constant $p M$, suppose that the period of the year $t$ is $n+1$, the calculation for purchase cost from the year $n T_{2}+1$ to $t$ is shown in formula (4).

$$
O C_{n+1}(r)=p q_{1}+p q_{2}+\cdots p q_{r}=\left\{\begin{array}{cc}
p M & T_{2} \geq r \geq T_{1} \\
p\left(q_{1}+q_{2}+\cdots q_{r}\right) & 0<r<T_{1}
\end{array}\right.
$$

Where $r=t-n T_{2}$.

The calculation for total purchase cost can be seen in formula (5).

b) Storage Cost

$$
O C(r)=\left\{\begin{array}{cc}
(n+1) p M & T_{2} \geq r \geq T_{1} \\
n p M+p\left(q_{1}+q_{2}+\cdots q_{r}\right) & 0<r<T_{1}
\end{array}, \quad r=t-n T_{2}\right.
$$

Suppose that the production cycle of equipment is $L$ years, the annual storage cost of a certain equipment is $h$ yuan, so the storage cost of the first year is $c 1=(1-L) h q_{1}$, the second year is $c 2=h q_{1}+(1-L) h q_{2}$, the third year is $c 3=h\left(q_{1}+q_{2}\right)+(1-L) h q_{3}$, and that is 
$c T_{1}=h\left(q_{1}+q_{2}+\cdots+q_{T_{1}-1}\right)+(1-L) h q_{T_{1}}$ in the year $T_{1}$.

Since the year $T_{1}+1$, the reserves remain at the level $M$, so the annual storage cost is a constant $h M$. Thus the calculation for storage cost in the first period can be seen in formula (6)

$$
\begin{aligned}
H C_{1} & =(1-L) h q_{1}+h q_{1}+(1-L) h q_{2}+h\left(q_{1}+q_{2}\right)+(1-L) h q_{3}+\cdots \\
& +h\left(q_{1}+q_{2}+\cdots+q_{T_{1}-1}\right)+(1-L) h q_{T_{1}}+\left(T_{2}-T_{1}\right) h M \\
= & (1-L) h M+\left(T_{2}-1\right) h q_{1}+\left(T_{2}-2\right) h q_{2}+\cdots+\left(T_{2}-T_{1}+1\right) h q_{T_{1}-1}+\left(T_{2}-T_{1}\right) h q_{T_{1}}
\end{aligned}
$$

The storage cost is a constant $T_{2} h M$ in the second period, the third period,..., and the period $n$. Formula (7) is the calculation for average reserves during $[t, t+T]$.

$$
\bar{M}=\frac{1}{T} \int_{t}^{t+T}\left[M-\frac{M}{T}(x-t)\right] d x=\frac{M}{2}
$$

The calculation for storage cost during this period is formula (8).

$$
\bar{M} h T=\frac{M}{2} h T
$$

So the storage cost is $H C_{n+1}(r)=r h M+\frac{M}{2} h T$ in $\left[n T_{2}+1, t+T\right]$, here $r=t-n T_{2}$, and the following is the same.

Formula (9) is the calculation for total storage cost.

$$
\begin{aligned}
& H C(r)=(1-L) h M+\left(T_{2}-1\right) h q_{1}+\left(T_{2}-2\right) h q_{2}+\cdots+\left(T_{2}-T_{1}+1\right) h q_{T_{1}-1}+\left(T_{2}-T_{1}\right) h q_{T_{1}} \\
& \quad+(n-1) T_{2} h M+r h M+\frac{M}{2} h T
\end{aligned}
$$

Formula (10) is the calculation for total cost.

$$
\begin{aligned}
T C(r)= & O C(r)+H C(r) \\
= & \begin{cases}(n+1) p M+(1-L) h M+\left(T_{2}-1\right) h q_{1}+\left(T_{2}-2\right) h q_{2}+\cdots & \\
+\left(T_{2}-T_{1}+1\right) h q_{T_{1}-1}+\left(T_{2}-T_{1}\right) h q_{T_{1}}+(n-1) T_{2} h M & T_{2} \geq r \geq T_{1} \\
+r h M+\frac{M}{2} h T & \\
n p M+p\left(q_{1}+q_{2}+\cdots q_{r}\right)+(1-L) h M+\left(T_{2}-1\right) h q_{1} & \\
+\left(T_{2}-2\right) h q_{2}+\cdots+\left(T_{2}-T_{1}+1\right) h q_{T_{1}-1}+\left(T_{2}-T_{1}\right) h q_{T_{1}} & 0<r<T_{1} \\
+(n-1) T_{2} h M+r h M+\frac{M}{2} h T & \end{cases}
\end{aligned}
$$

Because the occurred time $t$ of an emergency is uncertain, which can be regarded as random variable, if the probabilities of each year's emergencies are equal, so the random variable $r=t-n T_{2}$ meets the following equation: $P(r=1)=P(r=2)=\cdots=P\left(r=T_{2}\right)=1 / T_{2}$, to pursue the minimum expected cost $E(T C)$, which is shown in formula (11).

$$
\begin{aligned}
E(T C)= & T C(r=1) P(r=1)+T C(r=2) P(r=2)+\cdots+T C\left(r=T_{2}\right) P\left(r=T_{2}\right) \\
= & \frac{1}{T_{2}} \sum_{i=1}^{T_{2}} T C(r=i) \\
= & {\left[\left(T_{2}-1\right) h+\frac{p\left(T_{1}-1\right)}{T_{2}}\right] q_{1}+\left[\left(T_{2}-2\right) h+\frac{p\left(T_{1}-2\right)}{T_{2}}\right] q_{2} } \\
& +\cdots+\left[\left(T_{2}-T_{1}+1\right) h+\frac{p}{T_{2}}\right] q_{T_{1}-1}+\left(T_{2}-T_{1}\right) h q_{T_{1}}+\frac{M h T}{2}-\frac{\left(T_{1}-1\right) p M}{T_{2}} \\
& +(1+n) p M++(1-L) h M+(n-1) T_{2} h M+\frac{\left(1+T_{2}\right) h M}{2}
\end{aligned}
$$

Since the second period, the purchasing cost and storage cost of each period are constants $p M$ and $T_{2} h M$ respectively, which is not related to the purchase quantity, and the items that are not related to the purchase quantity in the above formulas are all ignored, so the objective function can 
be simplified as formula (12).

$$
\begin{gathered}
\min \left[\left(T_{2}-1\right) h+\frac{p\left(T_{1}-1\right)}{T_{2}}\right] q_{1}+\left[\left(T_{2}-2\right) h+\frac{p\left(T_{1}-2\right)}{T_{2}}\right] q_{2} \\
+\cdots+\left[\left(T_{2}-T_{1}+1\right) h+\frac{p}{T_{2}}\right] q_{T_{1}-1}+\left(T_{2}-T_{1}\right) h q_{T_{1}}
\end{gathered}
$$

The equivalent conversion from the minimization of objective function into the maximization of that can be seen in formula (13).

\section{2) Constraint Conditions}

$$
\begin{gathered}
\max -\left[\left(T_{2}-1\right) h+\frac{p\left(T_{1}-1\right)}{T_{2}}\right] q_{1}-\left[\left(T_{2}-2\right) h+\frac{p\left(T_{1}-2\right)}{T_{2}}\right] q_{2} \\
-\cdots-\left[\left(T_{2}-T_{1}+1\right) h+\frac{p}{T_{2}}\right] q_{T_{1}-1}-\left(T_{2}-T_{1}\right) h q_{T_{1}}
\end{gathered}
$$

The constraint condition $q_{i} \leq c^{i}$ is set due to the limitation in production capacity of equipment, to ensure the reserves can reach and remain at the level $\mathrm{M}$, it should meet $\sum_{i=1}^{T_{1}} q_{i}=M$.Based on the objective function and the constraint condition determined above, the linear programming model is established in formula (14).

$$
\begin{aligned}
& \max -\left[\left(T_{2}-1\right) h+\frac{p\left(T_{1}-1\right)}{T_{2}}\right] q_{1}-\left[\left(T_{2}-2\right) h+\frac{p\left(T_{1}-2\right)}{T_{2}}\right] q_{2} \\
& -\cdots-\left[\left(T_{2}-T_{1}+1\right) h+\frac{p}{T_{2}}\right] q_{T_{1}-1}-\left(T_{2}-T_{1}\right) h q_{T_{1}} \\
& \text { s.t. }\left\{\begin{array}{rl}
\sum_{i=1}^{T_{1}} q_{i}=M & \\
q_{i} \leq c^{i} & i=1,2, \cdots T_{1} \\
q_{i} \geq 0 & i=1,2, \cdots T_{1}
\end{array}\right.
\end{aligned}
$$

The stepwise purchase model when $T_{1}=T_{2}$.

When the purchasing time is equal to the lifespan of equipment, the stepwise purchase model is established, as shown in Figure 2.

When $T_{1}=T_{2}$, the annual purchase quantity of equipment is also a function with the period of $T_{2}$, in the interval of $\left[0, T_{2}\right]$.

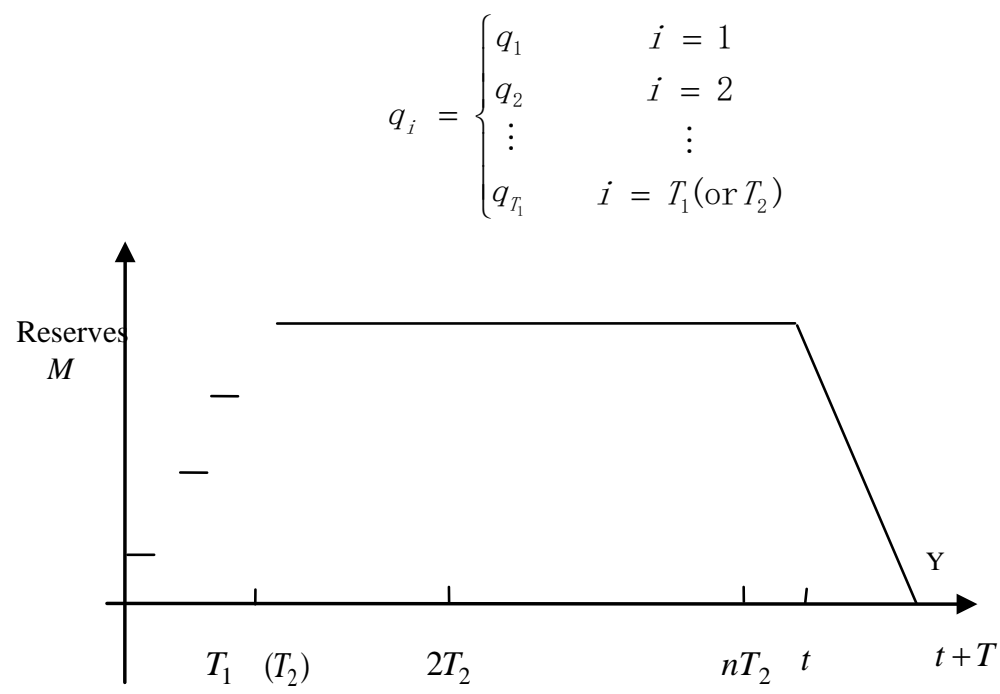

Figure 2.The relationship between the reserves and time when $T_{1}=T_{2}$ 
When $i>T_{2}, q_{i}=q_{r}$, where $r$ is a remainder that of $i$ divided by $T_{2}$.

The total purchase cost is $O C(r)=n p M+p\left(q_{1}+q_{2}+\cdots+q_{r}\right)$, here $r=t-n T_{2}$, and the following is the same.

Formula (15) is the calculation for total storage cost.

$$
\begin{aligned}
& H C(r)=(1-L) h M+\left(T_{2}-1\right) h q_{1}+\left(T_{2}-2\right) h q_{2}+\cdots+h q_{T_{1}-1} \\
& \quad+(n-1) T_{2} h M+r h M+\frac{M}{2} h T
\end{aligned}
$$

Formula (16) is the calculation for total cost.

$$
\begin{aligned}
T C(r)= & O C(r)+H C(r) \\
= & n p M+p\left(q_{1}+q_{2}+\cdots q_{r}\right)+(1-L) h M+\left(T_{2}-1\right) h q_{1} \\
& +\left(T_{2}-2\right) h q_{2}+\cdots+h q_{T_{1}-1}+(n-1) T_{2} h M+r h M+\frac{M}{2} h T
\end{aligned}
$$

Formula (17) is the calculation for expected cost.

$$
\begin{aligned}
E(T C)= & {\left[\left(T_{2}-1\right) h+p\right] q_{1}+\left[\left(T_{2}-2\right) h+\frac{p\left(T_{2}-1\right)}{T_{2}}\right] q_{2}+\cdots } \\
& +\left(h+\frac{2 p}{T_{2}}\right) q_{T_{1}-1}+\frac{p}{T_{2}} q_{T_{1}}+\frac{M h T}{2}+n p M++(1-L) h M \\
& +(n-1) T_{2} h M+\frac{\left(1+T_{2}\right) h M}{2}
\end{aligned}
$$

Formula (18) is the stepwise purchase model when $T_{1}=T_{2}$.

$$
\begin{gathered}
\max -\left[\left(T_{2}-1\right) h+p\right] q_{1}-\left[\left(T_{2}-2\right) h+\frac{p\left(T_{2}-1\right)}{T_{2}}\right] q_{2} \\
-\cdots-\left(h+\frac{2 p}{T_{2}}\right) q_{T_{1}-1}-\frac{p}{T_{2}} q_{T_{1}} \\
\text { s.t. }\left\{\begin{array}{c}
\sum_{i=1}^{T_{1}} q_{i}=M \\
q_{i} \leq c^{i} \quad i=1,2, \cdots T_{1} \\
q_{i} \geq 0 \quad i=1,2, \cdots T_{1}
\end{array}\right.
\end{gathered}
$$

The stepwise purchase model when $T_{1}>T_{2}$.

When the purchasing time is greater than the lifespan of equipment, the stepwise purchase model is established, as shown in Figure 3.

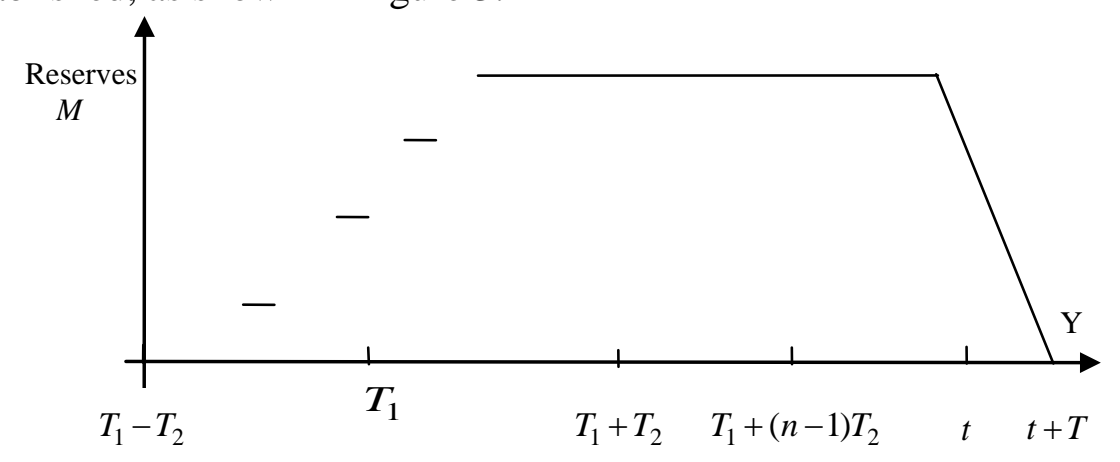

Figure 3.The relationship between the reserves and time when $T_{1}>T_{2}$

For the validity period $T_{2}<T_{1}$, in order to avoid the financial loss caused by equipment scrapping before the equipment inventory reaches $M$, the purchasing should be started in the year $T_{1}-T_{2}+1$, and the equipment reserves should reach $\mathrm{M}$ in the year $T_{1}$, after this the annual purchase volume will repeat with the period $T_{2}$. 


$$
q_{i}=\left\{\begin{array}{cc}
0 & 1 \leq i \leq T_{1}-T_{2} \\
q_{T_{1}-T_{2}+1} & i=T_{1}-T_{2}+1 \\
\vdots & \vdots \\
q_{T_{1}} & i=T_{1}
\end{array}\right.
$$

When $i>T_{1}, q_{i}=q_{T_{1}-T_{2}+r}$, where $r$ is a remainder that of $i-T_{1}$ divided by $T_{2}$.

Total purchasing cost: $O C(r)=n p M+p\left(q_{T_{1}-T_{2}+1}+q_{T_{1}-T_{2}+2}+\cdots+q_{T_{1}-T_{2}+r}\right)$, here $r=t-T_{1}-(n-1) T_{2}$, the following is the same.

Formula (19) is the calculation for total storage cost.

$$
\begin{aligned}
& H C(r)=(1-L) h M+\left(T_{2}-1\right) h h_{T_{1}-T_{2}+1}+\left(T_{2}-2\right) h q_{T_{1}-T_{2}+2}+\cdots+h q_{T_{1}-1} \\
& \quad+(n-1) T_{2} h M+r h M+\frac{M}{2} h T
\end{aligned}
$$

Formula (20) is the calculation for total cost.

$$
\begin{aligned}
T C(r)= & O C(r)+H C(r) \\
= & n p M+p\left(q_{T_{1}-T_{2}+1}+q_{T_{1}-T_{2}+2}+\cdots+q_{T_{1}-T_{2}+r}\right)+ \\
& (1-L) h M+\left(T_{2}-1\right) h q_{T_{1}-T_{2}+1}+\left(T_{2}-2\right) h q_{T_{1}-T_{2}+2} \\
& +\cdots+h q_{T_{1}-1}+(n-1) T_{2} h M+r h M+\frac{M}{2} h T
\end{aligned}
$$

Expected cost:

$$
\begin{aligned}
E(T C)= & {\left[\left(T_{2}-1\right) h+p\right] q_{T_{1}-T_{2}+1}+\left[\left(T_{2}-2\right) h+\frac{p\left(T_{2}-1\right)}{T_{2}}\right] q_{T_{1}-T_{2}+2}+\cdots } \\
& +\left(h+\frac{2 p}{T_{2}}\right) q_{T_{1}-1}+\frac{p}{T_{2}} q_{T_{1}}+\frac{M h T}{2}+n p M++(1-L) h M \\
& +(n-1) T_{2} h M+\frac{\left(1+T_{2}\right) h M}{2}
\end{aligned}
$$

Formula (21) is the stepwise purchase model when $T_{1}>T_{2}$.

$$
\begin{gathered}
\max -\left[\left(T_{2}-1\right) h+p\right] q_{T_{1}-T_{2}+1}-\left[\left(T_{2}-2\right) h+\frac{p\left(T_{2}-1\right)}{T_{2}}\right] q_{T_{1}-T_{2}+2} \\
-\cdots-\left(h+\frac{2 p}{T_{2}}\right) q_{T_{1}-1}-\frac{p}{T_{2}} q_{T_{1}} \\
\text { s.t. }\left\{\begin{array}{cl}
\sum_{i=1}^{T_{2}} q_{T_{1}-T_{2}+i}=M \\
q_{T_{1}-T_{2}+i} \leq c^{i} & i=1,2, \cdots T_{2} \\
q_{T_{1}-T_{2}+i} \geq 0 & i=1,2, \cdots T_{2}
\end{array}\right.
\end{gathered}
$$

\section{Model Solution}

\section{Simplex algorithm.}

Basic Thoughts: firstly, find out a basic feasible solution and determine whether it is the optimal solution, if not, then converting to the adjacent basic feasible solution, and increase the objective function value constantly until the optimal solution is found. The calculation steps are given below:

Step 1: figure out the initial basic feasible solution, and list the initial simplex tableau.

Step 2: optimality testing

If all the check numbers in the table meet $\sigma_{j} \leq 0$, and the nonzero artificial variables are not included in the basic variables, the basic feasible solution in the table is the optimal solution, however, there is no feasible solution when the basic variables contain the nonzero artificial variables, then the calculation is over. In the table, when $\sigma_{j}>0$, if the coefficient vector of the non-basic variable $x_{j}$ meets $P_{j} \leq 0$, so the problem has unbounded solutions, the calculation is 
over; otherwise, it will go to the next step.

Step 3.transform to another basic feasible solution which has a lager adjacent objective function value, list a new simplex tableau.

When the check numbers meet $\sigma_{j}>0$, finding out the biggest one $\sigma_{k}=\max _{j}\left\{\sigma_{j} \mid \sigma_{j}>0\right\}$, its corresponding variable $x_{k}$ can be regarded as the entering basic variable, the coefficient vector of $x_{k}$ meets $P_{k}=\left(a_{1 k}, a_{2 k}, \cdots a_{m k}\right), x_{l}$ is the leaving basic variable which is determined by the equation $\theta=\min \left\{\frac{b_{i}}{a_{i k}} \mid a_{i k}>0\right\}=\frac{b_{l}}{a_{l k}}$. A new basic feasible solution is found through replacing $x_{l}$ with $x_{k}$.

Step 4: repeat step 2 and step 3 until the end of the calculation.

\section{Two-phase Method.}

When solving the linear programming problem by simplex method, the initial basic feasible solution should be obtained firstly. The unit matrix is included in the coefficient matrix with constraint conditions after changing the linear programming problem into a standard form, with this as the initial basis, the initial feasible solution is obtained, and then the simplex method is used to get the solution. If there is no unit matrix included, artificial variables can be added to form a unit matrix, then we can obtain the initial feasible solution. But when using computer to get the solution, it is possible to make faults in computational results because of the incorrect value in calculation. In order to overcome this difficulty, the linear programming problem with artificial variables can be calculated in two phases.

The first phase of the two-phase method is to solve a linear programming problem only with artificial variables in its objective functions, namely, the coefficients of other variables in objective functions are set to 0 , and the coefficients of the artificial variables are set to a normal number (choosing 1 usually), the solution of the minimized objective function is obtained in the case of keeping the same constraint conditions of the original problems. Obviously in the first phase, when the artificial variable is set to 0 , the value of the objective function is also 0 .In this case, the optimal solution is a basic feasible solution of the original linear programming problem. In the first phase, if the objective function value of the optimal solution is not 0 , namely the nonzero artificial variables are included in the basic variables of the optimal solution, it shows that there is no feasible solution to the original linear programming problem. When the solving results of the first phase indicate that there is a feasible solution to the problem, the second phase is to remove the artificial variables in the original problem, and starting from this feasible solution (namely the optimal solution of the first phase), we continue to search for the optimal solution to the problem.

\section{Example Analysis}

Suppose that the demand of a certain kind of equipment is 13872 pieces, the unit price is 897 yuan, the lifespan is 15 years, the annual storage costs is 0.5 percent of the unit price, the production cycle is 4 months, the annual production capacity is 12000 pieces, the time can be used for production when encountering emergencies is 1.5 months, it will take 3 years to establish the reserves (suppose that the initial quantity of inventory is 0 ).

Due to the time can be used for production is less than the production cycle when encountering emergencies, so the reserves $M=13872$.

Because of $T_{1}<T_{2}$, a linear programming model is established according to formula (14).

$$
\begin{array}{r}
\max \quad-182.39 q_{1}-118.105 q_{2}-53.82 q_{3} \\
\text { s.t. }\left\{\begin{array}{cl}
q_{1}+q_{2}+q_{3}=13872 \\
q_{i} \leq 12000 \quad i=1,2,3 \\
q_{i} \geq 0 & i=1,2,3
\end{array}\right.
\end{array}
$$

It can be changed into standard form through adding slack variables and artificial variables, the two-phase method is used to get the following solutions. 
The linear programming problem in the first phase is:

$$
\begin{aligned}
& \max \quad-q_{7} \\
& \text { s.t. }\left\{\begin{array}{c}
q_{1}+q_{2}+q_{3}+q_{7}=13872 \\
q_{1}+q_{4}=12000 \\
q_{2}+q_{5}=12000 \\
q_{3}+q_{6}=12000 \\
q_{1}, q_{2}, \cdots, q_{7} \geq 0
\end{array}\right.
\end{aligned}
$$

The initial feasible solution $q^{0}=(0,0,0,12000,12000,12000,13872)$, iterative procedure with simplex method is shown in Table 1.

Table 1. Solving process with simplex method

\begin{tabular}{|c|c|c|c|c|c|c|}
\hline$c_{j}$ & -182.39 & -118.105 & -53.82 & 0 & 0 & 0 \\
\hline$c_{B}$ 基 $b$ & $q_{1}$ & $q_{2}$ & $q_{3}$ & $q_{4}$ & $q_{5}$ & $q_{6}$ \\
\hline$-182.39 \quad q_{1} \quad 12000$ & 1 & 0 & 0 & 1 & 0 & 0 \\
\hline $\begin{array}{lll}0 & q_{5} & 10128 \\
\end{array}$ & 0 & 0 & -1 & 1 & 1 & 0 \\
\hline $\begin{array}{lll}0 & q_{6} & 12000 \\
\end{array}$ & 0 & 0 & 1 & 0 & 0 & 1 \\
\hline$-118.105 \quad q_{2} \quad 1872$ & 0 & 1 & [1] & -1 & 0 & 0 \\
\hline$c_{j}-z_{j}$ & 0 & 0 & 64.285 & 64.285 & 0 & 0 \\
\hline $\begin{array}{lll}-182.39 & q_{1} & 12000 \\
\end{array}$ & 1 & 0 & 0 & 1 & 0 & 0 \\
\hline $\begin{array}{lll}0 & q_{5} & 12000\end{array}$ & 0 & 1 & 0 & 0 & 1 & 0 \\
\hline $\begin{array}{lll}0 & q_{6} & 10128 \\
\end{array}$ & 0 & -1 & 0 & {$[1]$} & 0 & 1 \\
\hline $\begin{array}{lll}-53.82 & q_{3} & 1872 \\
\end{array}$ & 0 & 1 & 1 & -1 & 0 & 0 \\
\hline$c_{j}-z_{j}$ & 0 & -64.285 & 0 & 128.57 & 0 & 0 \\
\hline $\begin{array}{lll}-182.39 & q_{1} & 1872 \\
\end{array}$ & 1 & {$[1]$} & 0 & 0 & 0 & -1 \\
\hline $\begin{array}{lll}0 & q_{5} & 12000 \\
\end{array}$ & 0 & 1 & 0 & 0 & 1 & 0 \\
\hline $\begin{array}{lll}0 & q_{4} & 10128 \\
\end{array}$ & 0 & -1 & 0 & 1 & 0 & 1 \\
\hline $\begin{array}{lll}-53.82 & q_{3} & 12000\end{array}$ & 0 & 0 & 1 & 0 & 0 & 1 \\
\hline$c_{j}-z_{j}$ & 0 & 64.285 & 0 & 0 & 0 & -128.57 \\
\hline $\begin{array}{lll}-118.105 & q_{2} & 1872 \\
\end{array}$ & 1 & 1 & 0 & 0 & 0 & -1 \\
\hline $\begin{array}{lll}0 & q_{5} & 10128 \\
\end{array}$ & -1 & 0 & 0 & 0 & 1 & 1 \\
\hline $\begin{array}{lll}0 & q_{4} & 12000\end{array}$ & 1 & 0 & 0 & 1 & 0 & 0 \\
\hline $\begin{array}{lll}-53.82 & q_{3} & 12000\end{array}$ & 0 & 0 & 1 & 0 & 0 & 1 \\
\hline$c_{j}-z_{j}$ & -64.285 & 0 & 0 & 0 & 0 & -64.285 \\
\hline
\end{tabular}

In table1, $c_{j}-z_{j} \leq 0$, and the artificial variables are not included in the basic variables, so the basic feasible solution $q=(0,1872,12000,12000,10128,0)$ in Table 2 is the optimum solution. Namely $q_{1}=0, \quad q_{2}=1872, \quad q_{3}=12000$

If the arrival period of this kind of equipment is 6 months, the annual purchase quantity and cost can be seen in Table 2 .

Table 2 Annual purchase quantity and cost of a certain equipment

\begin{tabular}{|c|c|c|c|c|}
\hline Year & $\begin{array}{c}\text { Annual purchase } \\
\text { quantity } q_{i}\end{array}$ & $\begin{array}{c}\text { Annual } \\
\text { purchase } \\
\text { cost }\end{array}$ & Annual storage fee & Total annual cost \\
\hline 1 & 0 & 0 & 0 & 0 \\
\hline 2 & 1872 & 1679184 & 4197.96 & 1683381.96 \\
\hline 3 & 12000 & 10764000 & 35305.92 & 10799305.92 \\
\hline 4 & 0 & 0 & 62215.92 & 62215.92 \\
\hline 5 & 0 & 0 & 62215.92 & 62215.92 \\
\hline$\ldots$ & $\ldots$ & $\ldots$ & $\ldots$ & $\ldots$ \\
\hline 15 & 0 & 0 & 62215.92 & 62215.92 \\
\hline
\end{tabular}

When $i>15, q_{i}=q_{r}, r$ is the remainder that of $i$ divided by 15 . 


\section{Conclusion}

Based on the stochastic process and optimization theory, the linear programming model for stepwise purchase of equipment is established, and the two-phase method which is more suitable for the computer solving is used to solve the model. This model can provide the theoretical basis and method for the formation of equipment purchase plan. Under the conditions of making full use of the limited purchasing funds and the maximum reduction in reserve management costs, a linear programming model is established and the two-phase method is used to solve the model based on the situation of the production and reserves. This method can avoid the computational errors caused by the false values in computer calculation, which is suitable for the computer solving. This model provides the theoretical method and computational model for the formation of equipment purchase plan.

\section{Reference}

[1] Ru Yihong,Tian Yuan. Logistics[M].Beijing. Higher Education Press.2009

[2] Xie Jinxing,Jiang Qiyuan,etc. Introduction to Linear Programming[M].Beijing. China Machine Press.2006

[3]Zhou Chao, Ma Honglu. The Research of Economical Stocks in Household Appliance Factory. Proceedings of the 2011 Conference on the Technology of China Household Electrical Appliances.2011

[4] Ren ChuangQi, Solving Linear Programming Problems in the Storage and Transportation by Algorithm Simplex Method. Contemporary Chemical Industry. Vol.42, No.2, 225-229, 2013

[5] ZHANG Yi. The unified approach of two-phase method and big M method. Journal of Shaanxi University of Technology (Natural Science Edition). No.2, 89-90, 2009 